\title{
Sugar Palm Fruit Galactomannan as an Alternative for Anti-Inflammation to Relieve Pain Intensity in Knee Osteoarthritis
}

\author{
Authors \\ Fatimah $^{1}$, Sugiyanto ${ }^{2}$, Donny Kristanto Mulyantoro ${ }^{3}$ \\ 1, 2, ${ }^{3}$ Politeknik Kesehatan Kemenkes Semarang \\ Jl. Tirto Agung, Pedalangan, Banyumanik, Semarang \\ Corresponding Author \\ Fatimah \\ Email: fatimah_yunaeza@yahoo.com
}

\begin{abstract}
Background: Osteoarthritis (OA) therapy uses non-steroids Anti-Inflammation Drugs (NSAID's), aspirin, and various analgesics. However, these may have side effects on the liver, kidney, gastrointestinal and interfere with the metabolism of cartilage. The previous study in animal showed induction of extract galactomannan of sugar palm fruit in Wistar rats reduced edema volume in pain arthritis compared to the control group. The carbohydrate content of sugar palm fruit mostly galactomannan (52.9\%) is a polysaccharide compound which has the function of analgesic and anti-inflammatory for pain.

Objectives: The purpose of this study is to evaluate sugar palm fruit as an alternative anti-inflammation to reduce pain intensity in osteoarthritis of the knee.

Methods: The study was carried out with the experimental method randomized pre test-post test control group design on 20 volunteers. There were three treatment groups and one control group. The control group, without administration of sugar palm fruit and three treatment groups with of sugar palm fruit; $1 x$ 50 gram in the morning; $2 \times 50$ grams in the morning and afternoon; and $3 \times 50$ grams in the morning, afternoon and evening; per days for seven days. Knee pain intensities were scored using a Western Ontario and McMaster Universities Osteoarthritis Index (WOMAC). Each group consist of Kellgren and Lawrence (KL) grade 0-4 of radiographic knee joint AP weight-bearing right and left. Data were analyzed using Paired T-Test and One Way ANOVA Test.

Results: Based on WOMAC score in pain, stiffness, and physical function respectively and overall showed that the intensity of knee pain per grading KL 0-4 against dose variation administration of sugar palm fruit there are significant differences between the control group with the treatment groups $(p=0.001)$. However, no significant differences among dose variation administration of sugar palm fruit for $1 x, 2 x$ and $3 x$ per day $(p>0.05)$.
\end{abstract}

Conclusions: Since there is no difference in dose variation, it is concluded that the most effective dosefeeding preparation is at $1 x$ collusion for the effective one is taken from the dose with the smallest frequency of administration.

Keywords: OA Knee, WOMAC, KL Grade, Galactomannan. 


\section{INTRODUCTION}

Osteoarthritis (OA) according to Academic College Of Rheumatoid (ACR), is a collection of conditions that affect the joints with signs and symptoms associated with the destruction of the integrity of arthritic cartilages (Puttini et.al 2005).

Osteoarthritis is a clinical syndrome due to structural changes in the joints and surrounding tissues characterized by progressive cartilage depletion accompanied by the new bone formation of the subchondral trabeculae and the formation of new bone at the edge of the joint (osteophytes). Histopathologically osteoarthritis is characterized by thinning of the cartilage along with bone growth and remodeling followed by atrophy and surrounding bone destruction, resulting in pain in the later stages leading to disability.

Western Ontario and McMaster Universities The Osteoarthritis Index (WOMAC) is one of the most common OA outcome instruments, especially in knee OA. WOMAC provides an algofunctional value that can be obtained through a questionnaire to measure joint pain and disability of patients knee OA. This instrument consists of 3 subscales of pain, stiffness, and limited physical function. On the pain sub-scale there are five questions about the intensity of pain felt on the joints, on walking, upstairs, rest, and at night. While the stiffness subscale consists of 2 questions about the severity of joint stiffness that is felt in the morning and afternoon / evening. In the sub-scale of the limitations of physical function, there are 17 questions. This sub-scale assesses the disability of knee OA sufferers who occur while going up and down stairs, standing from sitting, standing, bending to the floor, walking on flat surfaces, getting in / out of cars, shopping, wearing and removing socks, lying and getting out of bed, bathing, sitting, toilets, as well as doing the housework both light and heavy.

The imaging technique for detecting changes in the common joint structure undergoing $\mathrm{OA}$ is a clear radiographic photo of the knee. Plain photographs are still considered the gold standard for $\mathrm{OA}$ is diagnosing, thus, almost every service is available for X-rays for its relatively low cost, little checking times, relatively high resolution and non-invasive compared to other imaging techniques. Radiographic images have some limitations such as excellent radiographs for visualization of bone structure, but when looking at the joint prone can only be done by estimating the distance between the bony margins called the joint gap width or Joint Space Width (JSW). Another limitation is that the two-dimensional picture of radiography is unable to display all parts of the joint that are three-dimensional structures. Nevertheless, an assessment of knee radiographic images is still used as a gold standard, based on Kelgreen and Lawrence (KL) with a grading system based on two radiographic features: the presence or absence of osteophytes and Joint Space Narrowing (JSN). The knee radiograph is said to be OA when KL grade is $\geq 2$, while grade 0 and $1=$ average. Specifically, grade 2 = definite margin of osteofit, grade $3=$ observed JSN and grade $4=$ bone on bone contact (Kellgreen, 1957)

Sugar Palm Fruit (Indonesia $=$ Kolang-kaling) is oval, chewy and transparent white palm fruit seed with a refreshing taste. This fruit commonly used for beverage mixture is turned out to have significant benefits for stiff gestures namely as nutraceutical food that serves for good therapy for patients with osteoarthritis. Knee osteoarthritis usually causes pathological changes in joint structure, causing pain that leads to joint dysfunction, inflammation that appears at any time and disability. OA therapy generally uses NonSteroidal Anti-Inflammation Drugs (NSAID's), aspirin, and various analgesic drugs. However, these drugs have toxic side effects on the liver, kidneys, gastrointestinal and disrupt cartilage metabolism.

Commercial drugs have a gastrointestinal effect on rat digestion when tested while galactomannan extract has no effect. Therefore galactomannan extract found in sugar palm fruit can be used as an alternative to arthritis therapy.

\section{MATERIALS AND METHODS}

The design of this study was experimental with Pretest-Posttest Control Group Design. The 
subjects of the survey were 20 respondents. The group is divided into 1 control group, without given sugar palm fruit (X0) and 3 groups of treatment in the form of drinking sugar palm fruit; $1 \times 50$ gram in the morning (X1), 2 x 50 gram in the morning and afternoon (X2) and $3 \times 50$ gram in the morning, day and night (X3). Each treatment will be done for seven days. The study was based on an examination of knee intensity on the scale of Western Ontario and McMaster Universities Osteoarthritis Index (WOMAC). Radiographic imaging of osteoarthritis is assessed by KellgrenLawrence (KL) grade scales. Data are analyszed by using Paired T-Test and Wilcoxon Sign Test (bivariate) first, then continued with One Way Anova Test and Kruskal-Wallis Test (multivariate).

\section{RESULTS AND DISCUSSIONS RESULTS}

Respondents have recorded the gender, age, and Body Mass Index (BMI) as well as physical examination to know the symptoms and clinical signs of OA genu and intensity measurement of knee pain by filling the WOMAC (Pre-Test) questionnaire. Analysis of digital images of knee joints is performed in a standing position, projected AP weight bearing right and left. An assessment of the picture and degree of knee OA is carried out by a radiologist. In this study used respondents are the ones with digital radiographic images of KL grade $=0-4$.

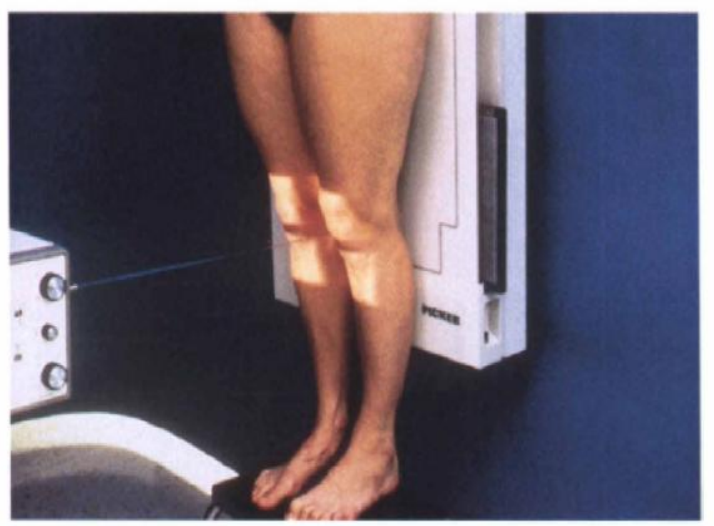

Figure 1. Projection of genu radiograph examination AP weight bearing right and left

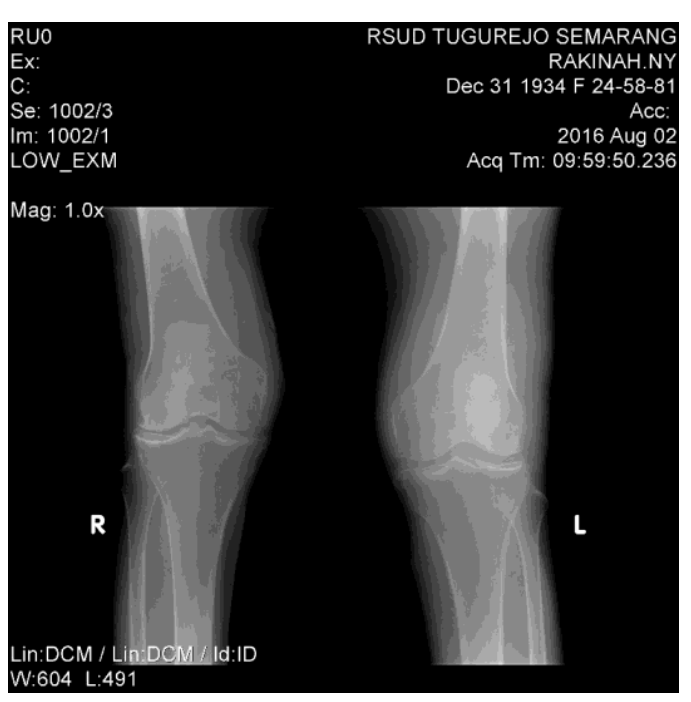

Figure 2. Results of digital images of genu radiographs AP weight bearing right and left

Giving Sugar Palm Fruit (Indonesia $=$ Kolangkaling) to the respondent was done for seven days, in 3 treatment groups with a dose of $1 \times 50$-gram morning (X1), 2 x 50-gram morning and afternoon (X2) and 3 x 50-gram morning, afternoon and night (X3). In the other hand, the control group is not given. After the drinking is completed, all respondents remeasured knee pain intensity by filling the WOMAC (Post-Test) questionnaire.

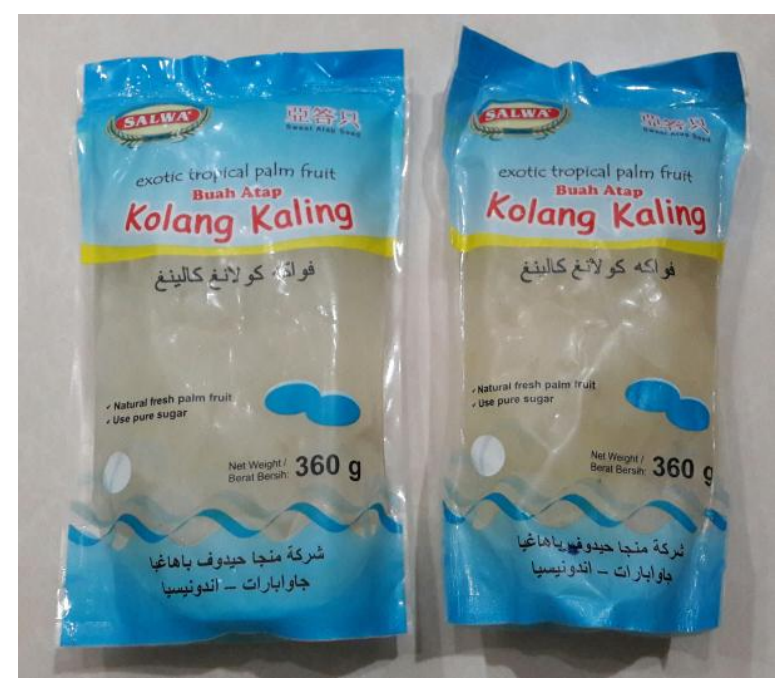

Figure 3. Sugar Palm Fruit (Indonesia $=$ Kolangkaling) Beverage

In the bivariate analysis WOMAC was performed per activity group (pain, stiffness and physical function) and as a whole against the control and treatment group (dose of $1 \mathrm{x}, 2 \mathrm{x}$ and $3 \mathrm{x}$ ). 
Table 1. Pre and post-Compare Means WOMAC results based on treatment group

\begin{tabular}{|c|c|c|c|c|c|c|}
\hline \multirow{3}{*}{$\begin{array}{l}\text { WO } \\
\text { MAC }\end{array}$} & \multicolumn{5}{|c|}{ Sugar Palm Fruit } & \multirow{3}{*}{$\mathrm{p}$} \\
\hline & \multirow{2}{*}{\multicolumn{2}{|c|}{ Control }} & Dose & Dose & Dose & \\
\hline & & & $1 \mathrm{x}$ & $2 \mathrm{x}$ & $3 \mathrm{x}$ & \\
\hline \multirow{2}{*}{ Pre } & 29.6 & \pm & $38.6 \pm$ & $40.8 \pm$ & $43.4 \pm$ & \multirow{2}{*}{0.866} \\
\hline & 21.51 & & 25.01 & 28.26 & 32.88 & \\
\hline \multirow{2}{*}{ Post } & & \pm & $32.4 \pm$ & $33.2 \pm$ & $26.6 \pm$ & \multirow{2}{*}{0.316} \\
\hline & 21.68 & & 21.31 & 23.86 & 20.27 & \\
\hline $\mathrm{p}$ & 0.000 & & 0.024 & 0.033 & 0.073 & \\
\hline \multirow{2}{*}{ Delta } & 22.4 & \pm & $-6.2 \pm$ & $-7.6 \pm$ & $-16.8 \pm$ & \multirow{2}{*}{0.001} \\
\hline & 3.78 & & 4.09 & 5.41 & 12.68 & \\
\hline
\end{tabular}

Table 2. Pre and post WOMAC test result between treatment group

\begin{tabular}{cccc}
\hline Variable & Dose 1x & Dose 2x & Dose 3x \\
\hline WOMAC Pre & \multicolumn{3}{c}{} \\
Control & 0.952 & 0.914 & 0.853 \\
Dose 1x & - & 0.999 & 0.992 \\
Dose 2x & & - & 0.999 \\
WOMAC Post & & & \\
Control & 0.505 & 0.539 & 0.291 \\
Dose 1x & - & 1.000 & 0.974 \\
Dose 2x & & - & 0.963 \\
WOMAC Gap & & & \\
Control & 0.001 & 0.001 & 0.001 \\
Dose 1x & - & 0.990 & 0.151 \\
Dose 2x & & - & 0.245 \\
\hline
\end{tabular}

Tables 1 and 2 show the overall WOMAC bivariate analysis in pain, stiffness and physical function, to the control and treatment group (dose of $1 x, 2 x$ and $3 x$ ). The result of the study shows the difference result of WOMAC significantly before and after given sugar palm fruit $(\mathrm{p}=0.001)$. However, there is no significant difference of WOMAC yield between treatment groups either $1 \mathrm{x}, 2 \mathrm{x}$ and $3 \mathrm{x}(\mathrm{p}>0,05)$.

\section{WOMAC Analysis on KL Grading to Control and Treatment Group}

The WOMAC bivariate analysis was also performed per grading KL $0-4$ to the control and treatment group (dose of $1 \mathrm{x}, 2 \mathrm{x}$ and $3 \mathrm{x}$ ).

Table 3. Comparing Means of pre and post Grade 0 based on treatment groups

\begin{tabular}{|c|c|c|c|c|c|}
\hline \multirow{2}{*}{$\begin{array}{l}\text { Grade } \\
0\end{array}$} & \multicolumn{4}{|c|}{ Sugar palm fruit } & \multirow[b]{2}{*}{$\mathrm{p}$} \\
\hline & Control & $\begin{array}{c}\text { Dose } \\
1 \mathrm{x}\end{array}$ & $\begin{array}{c}\text { Dose } \\
2 \mathrm{x}\end{array}$ & $\begin{array}{c}\text { Dose } \\
3 x\end{array}$ & \\
\hline Pre & $\begin{array}{c}1.67 \pm \\
1.53\end{array}$ & $\begin{array}{c}0.67 \pm \\
1.16\end{array}$ & $\begin{array}{c}0.33 \pm \\
0.58\end{array}$ & $0 \pm 0$ & 0.328 \\
\hline Post & $\begin{array}{c}8.33 \pm \\
5.86\end{array}$ & $\begin{array}{c}0.33 \pm \\
0.58\end{array}$ & $0 \pm 0$ & $0 \pm 0$ & 0.025 \\
\hline $\mathrm{p}$ & 0.252 & 0.317 & 0.317 & 1.000 & \\
\hline Delta & $\begin{array}{c}6.67 \pm \\
7.23\end{array}$ & $\begin{array}{c}-0.33 \pm \\
0.58\end{array}$ & $\begin{array}{c}-0.33 \pm \\
0.58\end{array}$ & $0 \pm 0$ & 0.042 \\
\hline
\end{tabular}

Table 4. Test Result of pre and post Grade 0 between group treatment

\begin{tabular}{cccc}
\hline Variabel & Dose 1x & Dose 2x & Dose 3x \\
\hline Grade 0 Post & & & \\
Control & 0.046 & 0.037 & 0.037 \\
Dose 1x & - & 0.317 & 0.317 \\
Dose 2x & & - & 1.000 \\
Grade 0 Gaps & & & \\
Control & 0.046 & 0.046 & 0.037 \\
Dose 1x & - & 1.000 & 0.317 \\
Dose 2x & & - & 0.317 \\
\hline
\end{tabular}

Tables 3 and 4 show the WOMAC bivariate analyses at grade 0 to the control and treatment groups (dose of $1 x, 2 x$ and $3 x$ ). The result of the analysis shows the significant difference between before and after giving sugar palm fruit $(\mathrm{p}=$ 0,042). However, there were no significant differences between treatment groups either dose $1 x, 2 x$ and $3 x(p>0,05)$.

Table 5. Comparing Means of pre and post Grade 1 based on treatment groups

\begin{tabular}{|c|c|c|c|c|c|}
\hline \multirow{2}{*}{$\begin{array}{l}\text { Grade } \\
1\end{array}$} & \multicolumn{4}{|c|}{ Sugar palm fruit } & \multirow[b]{2}{*}{$\mathrm{p}$} \\
\hline & Control & $\begin{array}{l}\text { Dose } \\
1 \mathrm{x}\end{array}$ & $\begin{array}{l}\text { Dose } \\
2 \mathrm{x}\end{array}$ & $\begin{array}{l}\text { Dose } \\
3 x\end{array}$ & \\
\hline Pre & $\begin{array}{ll}3.33 & \pm \\
2.08 & \end{array}$ & $8 \pm 7$ & $\begin{array}{l}8.67 \pm \\
5.69\end{array}$ & $\begin{array}{l}9.33 \pm \\
5.51\end{array}$ & 0.544 \\
\hline Post & $\begin{array}{l}12.33 \pm \\
11.02\end{array}$ & $\begin{array}{l}6.67 \pm \\
5.51\end{array}$ & $\begin{array}{l}7.33 \pm \\
5.13\end{array}$ & $\begin{array}{l}5.33 \pm \\
2.79\end{array}$ & 0.642 \\
\hline $\mathrm{p}$ & 0.244 & 0.270 & 0.05 & $0.057^{¥}$ & \\
\hline Delta & $9 \pm 9.54$ & $\begin{array}{l}-1.33 \\
\pm 1.53\end{array}$ & $\begin{array}{l}-1.33 \\
\pm 0.58 \\
\end{array}$ & $\begin{array}{l}-4 \pm \\
1.73\end{array}$ & 0.035 \\
\hline
\end{tabular}

Table 6. Test Result of pre and post Grade 1 between group treatment

\begin{tabular}{cccc}
\hline Variable & Dose 1x & Dose 2x & Dose 3x \\
\hline Grade 1 Gap & & & \\
Control & 0.050 & 0.046 & 0.046 \\
Dose 1x & - & 0.817 & 0.121 \\
Dose 2x & & - & 0.068 \\
\hline
\end{tabular}

Tables 5 and 6 show the WOMAC bivariate analysis in grade 1 to the control and treatment groups (dose of $1 x, 2 x$ and $3 x$ ). The result of the analysis shows the significant difference before and after sugar palm fruit feeding $(p=0.035)$ between control and treatment group of $1 \times(\mathrm{p}=$ $0,05), 2 x(p=0,46)$ and $3 x(p=0.046)$. However, there were no significant differences between treatment groups either dose $1 \mathrm{x}, 2 \mathrm{x}$ and $3 \mathrm{x}$ ( $\mathrm{p}>$ $0,05)$. 
The further test on Grade 2, Grade 3, and Grade 4 shows the similar tendency on the significant difference before and after sugar palm fruit feeding between control and treatment group but there were no significant differences between treatment groups either dose 1x, 2x and 3x.

\section{DISCUSSION}

The results of WOMAC knee pain intensity analysis performed both per WOMAC activity group (pain, stiffness and physical function) and WOMAC as a whole on control and treatment groups (doses $1 \mathrm{x}, 2 \mathrm{x}$ and $3 \mathrm{x}$ ), showed significant differences before and after the provision of sugar palm fruit.

In the control group, there were significant differences in each of WOMAC activity group (pain, stiffness and physical function) and overall WOMAC with mean pain intensity is greater than the treatment group. The pain is because of the control group did not get galactomannan intake for seven days.

In contrast to the treatment group (doses of $1 \mathrm{x}, 2 \mathrm{x}$ and $3 x$ ), the mean is smaller than the control group. In the treatment group dose of $3 x$ or larger means getting the more galactomannan intake, resulting in the lower value of means indicating the intensity of knee pain is decreasing. However, when tested the different means between treatment groups (giving the doses of $1 x, 2 x$ and $3 x$ ), the results were no significant differences. This way, when viewing from the aspect of the effectiveness of dose, the effective one according to the statistical results of the research is the administration of with a dose of $1 \mathrm{x}$ sugar palm fruit feeding.

WOMAC knee pain intensity analysis is performed by grading KL 0 - 4 to control group and treatment (doses of $1 \mathrm{x}, 2 \mathrm{x}$ and $3 \mathrm{x}$ ) where the result showed that at the grades 0,1 and 3 there were significant differences before and after administration sugar palm fruit. However, in KL grade 2 and 4 there was no significant difference.

In KL grades 0, 1 and 3 there are major differences between before and after treatment. In line with the WOMAC results, the mean pain intensity was greater in value for control group than the treatment group. This because the control group did not get the galactomannan intake like in the treatment group (doses of $1 \mathrm{x}, 2 \mathrm{x}$ and $3 \mathrm{x}$ ) making the value of the means are smaller indicating the intensity of knee pain is decreasing.

As previously discussed, the knee radiograph is said to be $\mathrm{OA}$ when grading $\mathrm{KL} \geq 2$, while grade 0 and $1=$ average. Grade $2=$ definite margin of osteofit, grade $3=$ observed JSN and grade $4=$ bone on bone contact (Kellgreen, 1957). As per grade assessment; 0 and 1 are normal or not OA making to respond to the galactomannan intake is still good. Grade $3 \mathrm{KL}$ is at OA position already with Joint Space Narrowing (JSN) observable. But in grade 3, OA symptoms are often stable, so the response to galactomannan is still good. This finding is by research conducted by Sharif et.al, (2011), where it was found that the progression of knee OA did not occur linearly.

In $\mathrm{KL}$ grade 2 and 4 there is no significant difference as in the grade 2, OA is a definite margin of osteofit while in grade 4 OA condition is getting worse happened bone on bone contact wherein both of these circumstances in taking of galactomannan do not show the effect of a significant change.

\section{CONCLUSIONS}

WOMAC knee pain intensity analysis was performed both on WOMAC activity group (pain, stiffness, and physical function) and as a whole and per grading KL 0 - 4 on variations of dose resulting in the significant difference between control and treatment groups. However, no significant differences in WOMAC outcome between treatment groups with the doses of $1 \mathrm{x}, 2 \mathrm{x}$ and $3 \mathrm{x}$. This way, it is inferred that the most effective dose for feeding preparation is $1 \mathrm{x}$ since the effective dose is taken from the dose with the lowest frequency of administration.

Based on the limitations encountered during the study, if research is conducted to assess the degree of severity of OA, early detection of OA, as well as for other purposes, should be performed by increasing the reliability of the assessment. The 
assessment should include the degree of

OA

severity knee and the exclusion criteria for patients suffering from OA outside the knee joint.

\section{REFERENCES}

1. Kellgren JH, Lawrence JS. Radiological Assessment Of Osteo-Arthrosis.Ann. rheum. Dis. 1957, 1, 16, 494.

2. Sarzi-Puttini, P., Cimmino, M. A., Scarpa, R., Caporali, R., Parazzini, F., Zaninelli, A., ... \& Marcolongo, R. (2005, August). Do physicians treat symptomatic osteoarthritis patients properly? Results of the AMICA experience. In Seminars in arthritis and rheumatism (Vol. 35, No. 1, pp. 38-42). WB Saunders.

3. Sharif, P. S., Abdollahi, M., \& Larijani, B. (2011). Current, new and future treatments of osteoporosis. Rheumatology International, 31(3), 289-300. 